\title{
DBT-A can enhance emotion regulation in ethnic minority youth
}

\author{
By Dr. Jessica K Edwards
}

Ethnic minority youth often experience environmental and culturally relevant stressors, putting them at risk of developing self-regulation difficulties and engaging in self-harm. ${ }^{1}$ Now, a pilot study conducted by researchers in New York provides preliminary evidence that dialectical behaviour therapy for adolescents (DBT-A) ${ }^{2}$ is associated with improved self-regulation skills in ethnic minority youth who self-harm.

Anna Yeo and colleagues found that upon completion of a 20-week DBT-A programme, 20 ethnic minority youth who displayed self-harming behaviours prior to treatment, had improved emotion regulation skills. They also found that baseline adaptive coping skills prior to treatment might predict post-treatment levels of DBT skills use. "Adolescents who exhibited low levels of adaptive coping skills prior to treatment, seemed to gain less DBT skills upon treatment completion", explains Yeo. "Thus, given that the application of DBT skills is a critical therapeutic milestone, clinicians should assess adolescents' baseline self-regulation skills prior to starting treatment and plan the focus and course of treatment accordingly".

The researchers propose that future randomized control trials should examine the effect of DBT-A on vulnerable ethnic minority youth's development of self-regulation. They also hope that more longitudinal research will explore how therapeutic changes in selfregulation might be linked to short-term and long-term clinical and functional outcomes in this population. "Improving self-regulation should be an essential intervention target when working with high-risk ethnic minority youth", says Yeo. "Pending future research, our study highlights potential utilities of DBT-A in enhancing self-regulation - a transdiagnostic factor associated with self-harm".

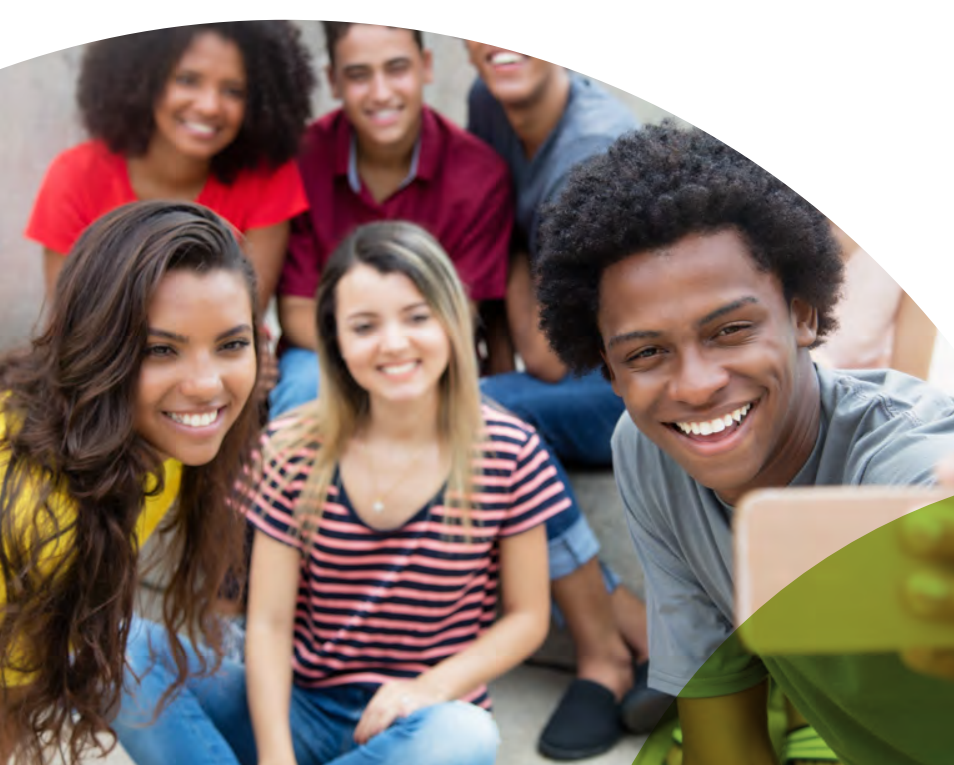

\section{Referring to:}

Yeo, A., German, M., Wheeler, L.A., Camacho, K., Hirsch, E. \& Miller, A. (2020), Self-harm and self-regulation in urban ethnic minority youth: a pilot application of dialectical behaviour therapy for adolescents. Child Adolesc. Ment. Health. doi: 10.111/camh.12374.

\section{References:}

${ }^{1}$ Cervantes, R.C. et al. (2014). Self-harm among Hispanic adolescents: Investigating the role of culture-related stressors. J. Adolesc. Health. 55:633639. doi: 10.1016/j.jadohealth.2014.05.017.

${ }^{2}$ Mehlum, L. et al. (2014). Dialectical behaviour therapy for adolescents with repeated suicidal and self-harming behaviour: A randomized trial. J. Am. Acad. Child Adolesc. Psychiatry. 53:1082-1091. doi: 10.1016/j.jaac.2014.07.003.

\section{Glossary:}

\section{Dialectical behaviour therapy for adolescents}

(DBT-A): a multi-component cognitivebehavioural treatment, in which the patient learns to manage difficult emotions by experiencing, recognizing and accepting them. DBT therapies use a balance of acceptance (accepting yourself as you are) and change (making positive changes in your life) techniques. Once the patient has learnt to accept and regulate emotions, they are then more able to change a harmful behaviour, such as self-harming. In general, DBT includes individual psychotherapy, family group skills training, telephone coaching and therapist team consultations.

Self-regulation: an individuals' efforts to alter their own inner states, responses, or behaviours including thoughts, emotions, impulses, and task performances.

Transdiagnostic: the transdiagnostic paradigm is founded on the concept that many mental health disorders share common etiological and maintenance processes. 